\title{
Contribution of the Nernst potential to stiffness constants: the asymmetrical case
}

S. Chatkaew and M. Leonetti ${ }^{\mathrm{a}}$

IRPHE, Université d'Aix Marseille I et II, CNRS, Technopole du Château Gombert, BP 146, 13384 Marseille Cedex 13 France Eur. Phys. J. E 17, 203 (2005)

Received 19 March 2007

Published online: 13 April 2007 - (c) EDP Sciences, Società Italiana di Fisica, Springer-Verlag 2007

The contribution of active membranes - described by a Nernst potential — to bending elastic modulus and to spontaneous curvature is determined and provided by equations (19) to (25) of reference [1]. A typing error (third term of the right member) appears in the first one, equation (19), that corresponds to the symmetrical case, the same Debye lengths inside and outside the vesicle.

$$
\kappa^{e f f}=\kappa+\frac{\left[\frac{k_{B} T}{e} \ln \left(\frac{n_{10}}{n_{1 \infty}}\right)\right]^{2}}{1+2 \epsilon_{m} / \epsilon d \chi}\left(\frac{\epsilon_{m} d}{12}+\frac{\epsilon_{m}^{2}}{12 \epsilon d^{2} \chi^{3}} \frac{9+12 d \chi+4 d^{2} \chi^{2}}{1+2 \epsilon_{m} / \epsilon d \chi}\right) .
$$

The numerical and graphical results are not affected by this error. The equations (20) to (25) are correct.

\section{References}

1. S. Chatkaew, M. Leonetti, Eur. Phys. J. E 17, 203 (2005)

\footnotetext{
a e-mail: leonetti@irphe.univ-mrs.fr
} 